\title{
Joint design standard for running times, dwell times and headway times
}

\author{
V. A. Weeda \& P. B. L. Wiggenraad \\ Department of Transport \& Planning, \\ Delft University of Technology, Netherlands
}

\begin{abstract}
A punctual train service requires a feasible timetable. This paper deals with design standards for running times, dwell times, and headway times. Feedback from operations to planning is an appropriate way to determine the required standards for delivering the desired performance. This feedback has been explored by analysing the traffic process in recent months on two Dutch mainlines. A conceptual conflict model has been developed to derive an empirical relationship between headways and reactionary delays. The operational observations make up the basis for a flexible joint standard for running, dwell and headway times. The suggested standard explicitly takes conflicts into account, either preventing them by scheduling a larger headway time, or compensating for them by increasing the running time supplement of the succeeding train.

Keywords: conflicts, punctuality, running time, timetable design.
\end{abstract}

\section{Introduction}

\subsection{Relevance}

Punctuality is an important performance indicator in railway transport. A punctual railway service requires both an adequate planning and operation close to the planning [1]. This paper deals with the first of these and focuses on three elementary processes in railway operations: running times, dwell times, and headway times. The timetable reserves a particular time laps for each process, consisting of two parts: the technically minimal time, and a margin to cope with variations during operations. A certain quantity of margins is necessary for a 
robust timetable; whether larger margins increase or decrease predictability is open to discussion. Clear is that too long margins raise production costs and, moreover, generate a less attractive railway service due to longer journey times or a limited train frequency. Well-considered allocation of margins could be done location-specifically, completely based on feedback from historical data. Although this method is very likely to produce a feasible timetable, it is quite an elaborate process that does not work in new situations. Therefore, this paper allocates margins by means of design standards. Determining the appropriate level of margins in these standards requires insight in two relationships:

- What is the quantitative relationship between margins and punctuality?

- As margins in running times, dwell times and headway times are all supposed to have a positive effect on punctuality, they should be interchangeable; according to which relationship?

\subsection{Research method}

These relationships have been investigated by statistical analysis of train running data and by analytical estimation of train delays. The railway traffic has been analysed in two case studies:

- Utrecht-Den Bosch, an electrified $48 \mathrm{~km}$ double-track mainline in the central part of the Netherlands, data from March 2004.

- Rotterdam-Dordrecht, an electrified $20 \mathrm{~km}$ four-track mainline in the West of the Netherlands, data from working days in November 2005.

These railway lines accommodate both inter-city services and local trains. Freight traffic runs on both lines as well, but this is not considered in the study.

Operational data are based on so-called TNV-logfiles: these files contain block section occupation and release data and other infra element status recordings from the Train Number Tracing system. The tool TNV-Prepare [2] extracts train runs and signal clearance times from these files. Recently, the logfiles have been enhanced with infrastructure data (the new recordings are called VTL-files). This format enables new functionalities for which the VTL-tool is developed (at NS Reizigers, the main Dutch passenger train operator). From measuring points at the front and end of a platform it calculates accurate arrival and departure times on the basis of actual rolling stock characteristics (train length, acceleration). It also determines unplanned stops in front of signals at danger.

Resulting train operation data have been broken down by type of rolling stock and by time of day. This decomposition yielded some minor differences but these do not influence the findings given in this paper and are not issued there.

\subsection{Paper outline}

The paper is structured as follows. Sections 2, 3 and 4 deal with running times, dwell times and headway times, respectively. These sections start with a short description of the current design standard; consequently, results of the operational analysis are presented. Section 5 gives an integrated view on the three types of processes and suggests a joint standard. Finally, section 6 draws conclusions. 


\section{Running times}

\subsection{Current standard}

In current Dutch planning practice running times are calculated microscopically on the basis of infrastructure and rolling stock characteristics. On top of that, the planning standard prescribes a 7\% supplement. This is supposed to cover daily variations in running times due to driving behaviour, weather circumstances, train load, etc. This standard is a minimum; capacity constraints and network coherence may force planned running times to be longer.

\subsection{Operational practice}

The feasibility of running times planned according to the standard has been assessed on the basis of autonomous runs, i.e. trains not held up by restrictive signals. Delays as a result of congestion effects are addressed separately in section 4. With help of signal status information in TNV data, perturbed runs can be identified and filtered. This filtering is reasonably accurate, but some cases of reactionary delays are still in the dataset, including unplanned stops at (not recorded) permissive signals.

The Utrecht-Den Bosch case study showed that inter-city trains make their runs within schedule, whereas local trains did not manage to do so. The next question was if the unfeasibility of local trains' paths was caused by running times, dwell times, or both. The relevant data, however, was too rough to isolate dwellings from running times. The Rotterdam-Dordrecht case study made this distinction possible. Apart from freight and international traffic, this line accommodates per hour per direction 4 inter-city services (running time approx. 14 minutes) and 6 local services with intermediate stops (running time 18-24 minutes).

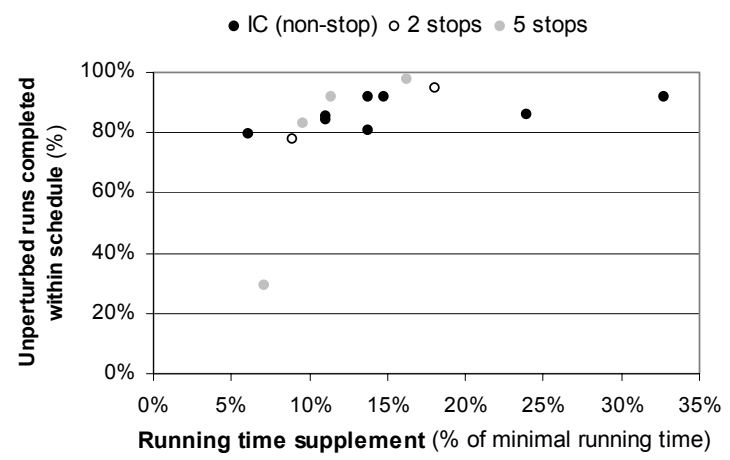

Figure 1: Feasibility of planned running times for autonomously running trains per train series on Rotterdam-Dordrecht. 
Figure 1: displays for each train series the percentage of runs completed within scheduled running time, depending on the available running time supplement. Like between Utrecht and Den Bosch, a 7\% supplement is already sufficient for about $80 \%$ of the unperturbed inter-city trains; a (much) larger supplement does not improve performance. Demanding a 100\% running time feasibility is not necessary, taking into account that the dataset may still include conflicting runs or occasionally delays due to special causes along the way.

For local trains, the picture is slightly different. Supplements over $10 \%$ produce a feasible running time, but a path with only $7 \%$ appears to be too tight and can only be adhered by $30 \%$ of the trains of the series. Given the data volume and accuracy, this is definitely not an outlier. To find an explanation why a 7\% supplement is enough for inter-city trains but not for local trains, the next paragraph looks into at the calculation of supplements.

\subsection{Basis for running time supplements}

Traditionally, the length of running time supplements is determined as a percentage of the minimal running time. This is not obvious, however. It is essential to realise that factors such as weather, train load and driving behaviour generally influence acceleration rather than top speed. The case study data shows that variations in running times are high in the accelerating and braking areas and much lower in the cruising area in-between. Hence, the need for supplements especially exists around stops rather than equally distributed along the way. Remarkably, local trains, stopping more frequently than inter-city trains, have no extra supplement but $7 \%$ of the running time. To this end, it seems logical to base the supplement on the number of stops instead of the minimal running time. This idea is worked out in section 5 .

\section{Dwell times}

\subsection{Current standard}

Technically minimal dwell times are not as easy to calculate as minimal running or headway times. In a way, the minimal dwell time is just the time required to open and close the doors. Practically, the dwell time largely depends on the (widely varying) number of boarding and alighting passengers. Dwell times are therefore scheduled in different ways depending on station importance:

- Depending on passenger numbers main stations have a minimal dwell time of 1,2 or 3 minutes for inter-city trains. In the same station categories the standards for local trains are 1,1 and 2 minutes, respectively.

- At line-side stops, the minimal dwell time varies from 0.5 to 0.7 minute, determined by the type of rolling stock (depending on door width and spacing, amongst other factors). In the near future, a 1 minute dwell time will be required at a couple of busy line-side stops. As a rule, departure times are rounded down to avoid waiting and wasting time.

In planning dwell times, the concept of margins is not used explicitly. 


\subsection{Operational practice}

Just as the running time analysis, the dwell time analysis focuses on RotterdamDordrecht. The dwell time analysis analogously excluded stops were the train had to wait for the departure signal to turn clear. Early arriving trains have been removed from datasets as well, as they have to await scheduled departure and thus are not representative for minimal dwelling.

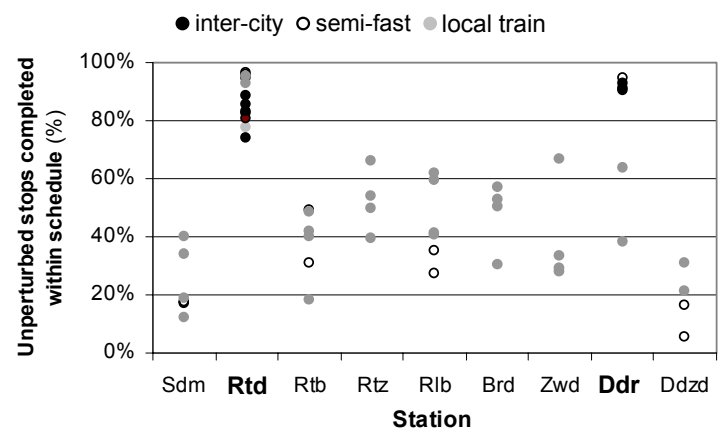

Figure 2: Feasibility of planned dwell times for autonomous stops per train series on Rotterdam-Dordrecht.

Figure 2: features the percentage of trains in a series that meets the scheduled dwell time per station. Stops exceeding the standard are left out. The remaining stops are scheduled at 2 minutes at Rotterdam (Rtd) and Dordrecht (Ddr), but 1 minute for local trains at Ddr, and 0.6 or 0.7 minute at line-side stops. The picture is clear:

- Dwell times at main stations are feasible for all inter-city trains. The 1 minute standard for local trains (Ddr) is questionable: the operational average exceeds the standard.

- Dwell times at line-side stops are unrealistic. At some spots they are even very unrealistic, only being met by about $30 \%$ of the trains.

\section{Headway times}

\subsection{Current standard}

In this project, the term "headway" applies to any situation where two trains consequently use the same infrastructure (track, switch or crossing). In contrast with running times, headway times are in the Netherlands planned in a macroscopic way. Generally, the minimal interval between two train movements at the same location is 3 minutes, although other (integer) headway times hold for certain types of successions and for certain locations. The difference between the technically minimal headway time and the planned interval is available as buffer time, allowing a train to run slightly late without consequences for other trains. 


\subsection{Conceptual model infrastructure conflict}

If a train's delay exceeds the buffer time, it may cause reactionary delays to succeeding trains. This mechanism has been studied with a newly developed conceptual model, which will be enlightened with an example from the UtrechtDen Bosch case study.

The situation is a level junction where two consecutively train movements ("train 1" and "train 2") have a crossing in common. Their scheduled headway time is 3 minutes with 44 seconds of buffer time. Before the trains meet and possibly influence each other, both of them have a certain deviation from the timetable (positive, zero or negative). These deviations are derived from the TNV data and plotted against one another in Figure 3:. Each of these approx. 700 dots represents one couple of train 1 and train 2 . For every dot, it is determined whether train 1 held up train 2. A few conditions divide the dots into a few categories:

Ia Train 1 is running on time or has a delay smaller than the buffer time. Train 2 is on time or late and is not perturbed (40\%).

Ib Train 2 is running early and is held up (under the diagonal dotted line). It does not matter since it only makes train 2 run on time again $(16 \%)$.

Ic Train 1 is late but train 2 is even later and does not suffer an additional delay. Although train 2 should not run late, there is no headway conflict $(12 \%)$.

II Train 2 reaches the junction before it is released by train 1. A conflict occurs: train 1 delays train 2 or occasionally the other way round $(23 \%)$.

III Train 1 is running that late, that the train dispatcher gave priority to train $2(9 \%)$.

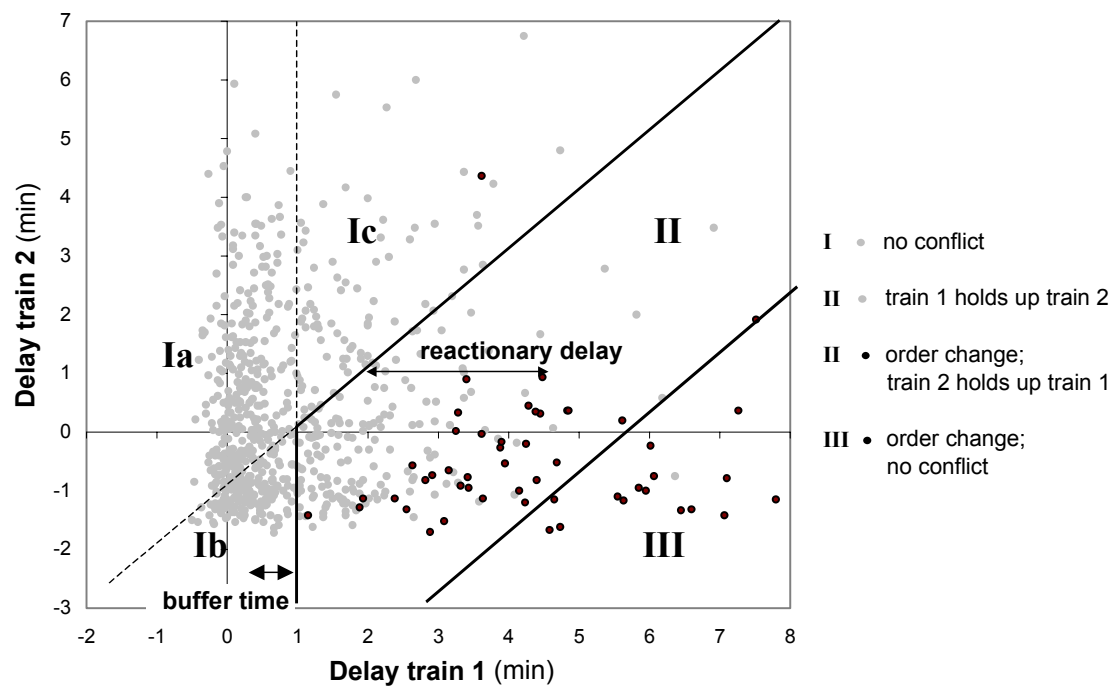

Figure 3:Determining reactionary delay from initial delays train 1 and train 2 . 
The observed percentage of reactionary delayed trains can well serve as an estimation of the probability of conflicts in the unchanged future situation. Figure 3: also gives an estimation of the time a train loses compared to an unperturbed run. The reactionary delay of a perturbed train 2 can be calculated as the horizontal distance between a category II dot and the left border line of category II:

$$
r_{2}=d_{1}-\max \left(0, d_{2}\right)-b_{12}
$$

with:

$r_{i}$ reactionary delay to train $i$

$d_{i}$ initial delay to train $i$

$b_{i j} \quad$ buffer time between train $i$ and $\operatorname{train} j$

In category II, both trains approach the junction at the same time. A conflict for either train is then inevitable. In case of train order change, the reactionary delay of train 1 can be calculated analogously to (1) as the horizontal distance to the right diagonal line. This delay is not considered here because its size is negligible for the entirety of "trains 1". In case both trains come from the same track, order change is no option at all.

\subsection{Relationship buffer time - reactionary delay}

All calculations so far were observations, but the model is suitable to make predictions as well. Again, the conflict at Den Bosch serves as an example. Suppose that the departure of train 1 is put forward by one minute. This earlier departure enlarges the probability that train 1 has released the junction when train 2 is coming. Now, two assumptions are made:

- The delay distributions are independent. This only holds if the involved trains come from different tracks. If the trains have been following each other, train 1 may have been holding up train 2 before.

- The delay distributions stay constant. This is imaginable if the entire path of train 1 is advanced by one minute. Then, all "trains 1" depart one minute earlier from Den Bosch than they did in the timetable 2004.

Separating trains in time by one more minute corresponds with adding one minute of buffer time. Current buffer time can be seen left at the bottom in Figure 3:. By increasing this distance (Figure 4:: shifting the diagonal lines horizontally), the effect of a longer buffer time can be determined:

- Category I widens, i.e. a larger number of "trains 2" will not suffer any reactionary delay.

- The reactionary delay of perturbed trains (category II) will fall.

- The number of order changes will decrease; in some category II cases this will create a delay for train 2 . In category II the model assumes that the train dispatcher always chooses the order with minimal total delay. 


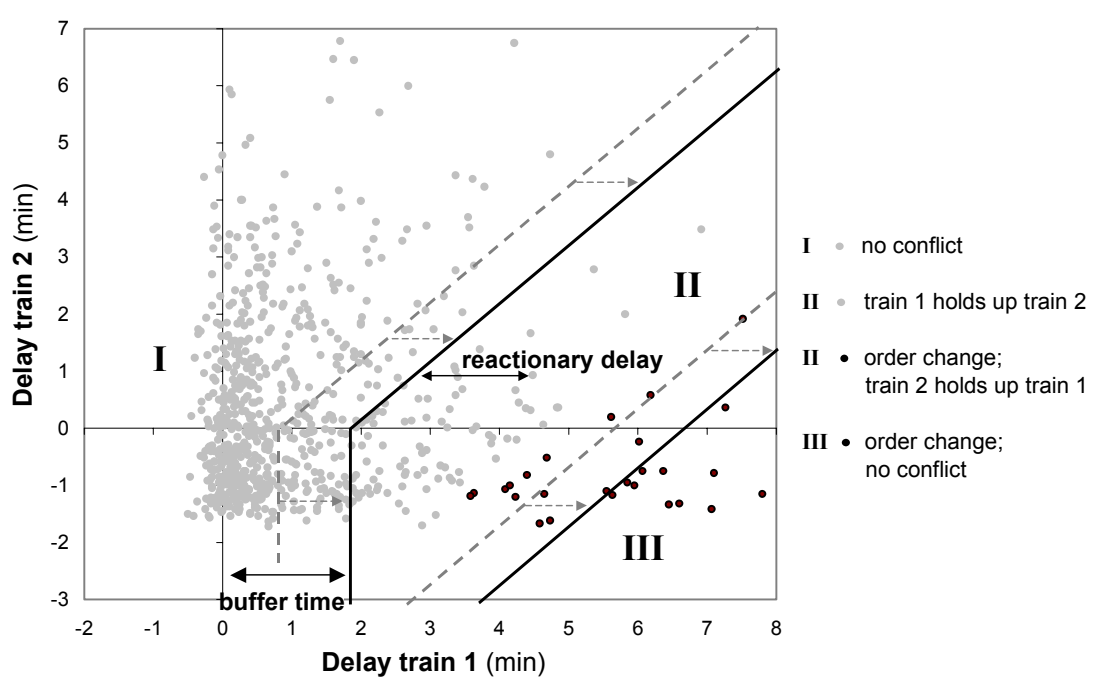

Figure 4: Determining reactionary delay at changing buffer time.

On the basis of these new figures, the average reactionary delay of all "trains 2 " can be calculated. This was done for every value of the buffer time. This yields a relationship between buffer time and reactionary delay (see Figure 5:, the black curve). Such a calculation was carried out for other short scheduled train successions in the case studies; each curve in the figure represents the relationship of a specific conflict. Although the reactionary delay depends highly on the delay distributions of both trains, the curves look similar: buffer time limits reactionary delay, but the benefits decrease with increasing buffer time. With a buffer time over 2 minutes the average reactionary delay becomes negligible.

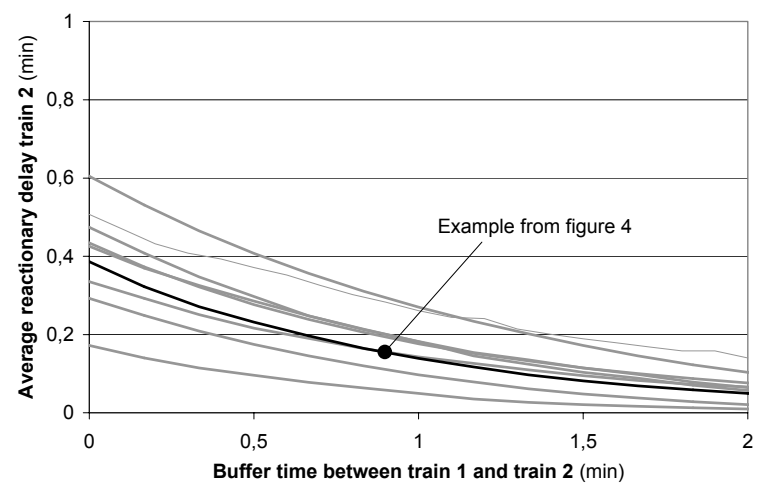

Figure 5: Calculated relationship between buffer time and reactionary delay. 


\section{Joint standard}

Robust design standards should basically enable traffic to run smoothly under daily stochastic conditions. Following that principle this section will stepwise integrate running, dwell, and headway times toward a joint design standard.

\subsection{Integration of running and dwell times}

In sections 2 and 3 it was found that inter-city train's running times and dwell times at main stations are feasible. Local trains' paths, however, were shown to be too tight. This difference is caused by two elements:

- Variation in running times occurs around stops, of which local trains have more.

- The standard for line-side stops is too short.

These problems are tackled with two changes in the standards. First, equally distributed supplements (the current 7\%) are replaced by a fixed supplement per main station stop (or: per node-to-node run) to cover acceleration and braking variation. The empirical data of inter-city trains indicates that 1.5 minutes per stop is sufficient for a running time feasible for most trains (over $75 \%$ of the unperturbed runs of all series in both case studies). To make a practicable standard, this value is also used for local trains per main station stop.

For local trains, secondly, line-side stops and running time variations around them are addressed in connection. The data shows that both components together require an extra 0.4 minute per stop, on top of the 1.5 minutes mentioned above. An easy way to include that time in the train path is adding it to the dwell time at line-side stops: it does not matter whether margins are put in running times or in dwell times, whenever the sum of both is large enough. With a current standard of 0.6 minute on average this makes up a new dwell time of 1.0 minute.

\subsection{Integration of running and headway times}

The previous paragraph gave an integrated approach for running and dwell times. With that, feasible paths can be planned for autonomously running trains. When multiple trains run on a network, they may interfere and cause conflicts. Here headway times show up. The idea is this:

- Conflicts are prevented with buffer times between train paths.

- The reactionary delay still occurring is cured with an extra running time supplement for the succeeding train. This supplement is equal to the expected average delay, according to Figure 5:.

When consistently network-wide implemented, this regime will generate a planning which is robust against daily variations. A usual requirement for robustness is a not-increasing average delay [3] and that is achieved with this standard. The quantified interchangeability of different types of margins provides flexibility in timetable design. An appropriate combination of running time and headway time should be chosen situation-specifically. 


\subsection{Overall integration}

On the basis of the steps described above, a joint design standard for running, dwell and headway times is suggested (under other circumstances, values may differ but the concept still holds):

- Between two main nodes trains (both inter-city and local ones) have a fixed supplement of 1.5 minutes covering acceleration and braking variations around the nodes, on top of the minimal running time.

- The dwell time standard at line-side stops is raised from 0.5-0.7 minute to 1.0 minute in order to cover excessive dwelling and varying driving behaviour around these stops. Dwell times at the nodes stay as they are.

- At short headways, a succeeding train gets an additional running time supplement depending on the buffer time between the train movements, compensating for reactionary delay. This supplement varies from 0.4 minute in case of no buffer to zero in case of 2.0 minutes buffer time.

\section{Conclusions}

A railway timetable needs certain margins in order to deal with operational variation. Computer tools enable detailed analysis of train running data, which shows that design standards for local services are too tight and that current planning practice insufficiently takes headway conflicts into account.

An alternative joint design standard is proposed, no longer determining running time supplements as a percentage of minimal running times, but allocating them where they are needed: depending on the number of stops. In conjunction, dwell times at line-side stops are extended. Conflicts between trains can be either prevented by separating paths with buffer times or cured by an additional supplement in the succeeding train's running time, compensating the expected reactionary delay. The suggested standard would improve punctuality of the railway service and flexibility of the timetable design process.

\section{Acknowledgement}

This project has been performed within the TRANSUMO program "Reliability of transport chains", funded by Dutch government and in co-operation with ProRail.

\section{References}

[1] Fénix, J., "User centred design applied to increase timetable stability". Accepted for the 36. Tagung Moderne Schienenfahrzeuge, Graz, Sept. 2005

[2] Goverde, R.M.P. en I.A. Hansen, "TNV-Prepare: Analysis of Dutch railway operations based on train detection data". In: J. Allen et al, Computers in Railways VII, WIT Press, Southampton, 2000, pp. 779-788

[3] Union International des Chemins de fer, Capman Working Group, Capacity Management (Capman Phase 3). Summary report, Dec. 2004 